\title{
Lupus Anticoagulant Increases Activated Partial Thromboplastin time (PTT) Prolongation in Incubated 1:1 Mix
}

\section{GRACE MONIS, CHRIS FERRELL, MORAYMA REYES}

\begin{abstract}
Our study analyzes the effects of incubation time and strength of lupus anticoagulant (LAC) on clotting times and prolongation of activated partial thromboplastin time (PTT) 1:1 mix assays with incubation. The prolongation in seconds of PTT 1:1 mix after incubation in the confirmed presence or absence of LAC was correlated to strength of the LAC as well as length of incubation (1 vs. 2 hours).
\end{abstract}

Our study suggests that when screening for possible Factor VIII (FVIII) inhibitors, a 2 hour incubation of a PTT 1:1 mix increases the frequency of false positives as compared to 1 hour incubation, and that most of these false positives are due to LACs. Prolongation of clotting times for PTT 1:1 mixes in patients with LAC is influenced by both length of incubation time and strength of the LAC. Conclusions: When using PTT 1:1 mixes to screen for FVIII inhibitors, the effect of a possible LAC on the interpretation of the PTT prolongation should be considered. This effect is influenced by both incubation time and LAC strength.

\begin{abstract}
ABBREVIATIONS: ANOVA - analysis of variance; BLUP - borderline LAC; FVIII - Factor VIII; IgA immunoglobulin A; IgG - immunoglobulin G; IgM immunoglobulin M; LAC - lupus anticoagulant; LIS laboratory information system; MLUP - moderate LAC; NEG - negative; PTT - activated partial thromboplastin time; PT - prothrombin time; SLUP - strong LAC; SEM - standard error of the mean
\end{abstract}

INDEX TERMS: Lupus Anticoagulant, Lupus Inhibitor, Factor VIII Inhibitor, Factor VIII, 1:1 mix, PTT

Clin Lab Sci 2012;25(3):165

Grace Monis, MD-PhD, University of Washington
Laboratory Medicine, Seattle, WA

Chris Ferrell, MT(ASCP), Harborview Medical Center, Seattle, WA

Address for Correspondence: Morayma Reyes, MDPhD, 815 Mercer Street Room 332, Box 358050, Seattle, WA 98195, 206-616-5004, morayma@u.washington.edu

\section{INTRODUCTION}

A 23 year old man with Bechet's disease was status post superior mesenteric aneurysm repair and had a prothrombin time (PT) of 13.2 seconds (normal range $=10.7-15.6$ seconds) and PTT of 50 seconds (normal range $=22-35$ seconds). Bechet's disease is an inflammatory, multi systemic disease of unknown etiology that may present with spontaneous remissions and relapses. Progression of the disease may lead to organ damage, including characteristic oral and genital mucous ulcers, uveitis and dermic lesions. Histopathologically, Bechet's is characterized by a systemic vasculitis with perivascular inflammatory infiltrates. ${ }^{1}$ On 1:1 mix, the patient's PTT immediately corrected to 34 seconds (control PTT was 28 seconds) (Table 1). However, after a 1 hour incubation of the 1:1 mix, the patient's PTT was 41 seconds (control PTT was 30 seconds). This 7 second prolongation raised concern about the possibility of a Factor VIII (FVIII) inhibitor (Table 1). A chromogenic FVIII assay was performed which indicated an activity of $228 \%$ with no inhibition. A hexagonal phase lupus anticoagulant assay revealed a moderate strength lupus anticoagulant.

Lupus Anticoagulants (LAC), also known as Lupus inhibitors, lupus-like inhibitors and anti-phospholipid antibodies are antibodies that compete with other proteins in the coagulation cascade for phospholipid. This interference results in prolongation of coagulation clotting assays, primarily the activated partial 
thromboplastin time (PTT), but prolongation of the PT has also been reported. ${ }^{2}$ LACs may therefore complicate interpretation of clotting based assays and screens, such as FVIII inhibitor detection. ${ }^{3}$ LACs are often IgG but can also be $\operatorname{IgM}$ or IgA. ${ }^{4}$ They may be seen in patients with immunological disorders or transient inflammatory conditions. LAC interference is phospholipid dependent. It is postulated that, in vivo, LACs bind to both phospholipid and another protein

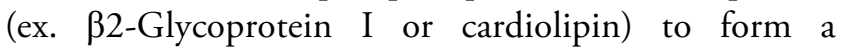
complex that may activate platelets or vessel endothelium leading to a pro-thrombotic microenvironment. ${ }^{5}$

Table 1. In our laboratory, PTT prolongations are calculated by subtracting the patient's immediate 1:1 mix PTT from the patient's incubated 1:1 mix PTT (middle column). A prolongation of 6-7 seconds (borderline) or $\geq 8$ seconds (positive) in the incubated 1:1 mix PTT was the criteria used to screen for possible FVIII inhibitors; most specimens were then retested by a FVIII inhibitor Bethesda assay or a chromogenic FVIII assay. Results of the screen are acceptable as long as control (normal pool) plasma PTT prolongation with incubation was less than 3 seconds.

\begin{tabular}{|c|c|c|c|}
\hline & $\begin{array}{l}\text { Patient PTT } \\
\quad(\mathrm{sec})\end{array}$ & $\begin{array}{c}\text { Patient PTT } \\
\text { 1:1 mix (sec) }\end{array}$ & $\begin{array}{c}\text { Control (Pool) } \\
\text { PTT (sec) }\end{array}$ \\
\hline Immediate & 50 & 34 & 28 \\
\hline Incubated ( $1 \mathrm{hr}$ & 61 & 41 & 30 \\
\hline Prolongation & 11 & 7 & 2 \\
\hline
\end{tabular}

When a patient has a prolonged PTT, one way to differentiate between the presence of an inhibitor and a factor deficiency is by exploiting the interference of the PTT in a 1:1 mix. ${ }^{6}$ A PTT 1:1 mix is performed using equal portions of patient plasma and normal pooled plasma. If correction on this immediate PTT mix is incomplete (result does not return to the normal PTT reference range), then prolongation due to an inhibitor is likely. Complete correction of the PTT to within the normal reference range usually indicates the presence of a factor deficiency. However, FVIII inhibitors, considered slow acting inhibitors, may also show complete correction on the immediate mix. To screen for the presence of a FVIII inhibitor, a 1 to 2 hour incubation at $37^{\circ} \mathrm{C}$ is performed on the $1: 1 \mathrm{mix}$, and the PTT 1:1 mix result after incubation is compared to the immediate PTT 1:1 mix result. In our lab, a prolongation of $\geq 8$ seconds in the incubated sample is suggestive of a FVIII inhibitor while prolongation of 6-
7 seconds may be considered "borderline" prolongation. If an inhibitor is suspected, the presence of either a factor specific inhibitor or LAC is then ascertained with a confirmatory assay such as Bethesda or hexagonal phase phospholipid assay respectively. ${ }^{7}$ Using a PTT 1:1 mix is an efficient and cost effective way to screen for inhibitors before investing time, effort and resources in performing a Bethesda assay or LAC assay.

LACs are commonly thought to be immediate acting inhibitors, causing incomplete correction in the immediate PTT 1:1 mix. However, atypical LACs have been reported which behave more like a slow acting inhibitor, as was demonstrated in the case history above. In addition, when our laboratory increased the 1:1 mix incubation time from 1 hour to 2 hours in an attempt to increase sensitivity to FVIII inhibitors, we began noticing an increased incidence of false positive prolongations of incubated 1:1 mixes (i.e. prolongation was not due to FVIII inhibitor). To better understand the behavior of LACs and the effect they may have on interpretation of clotting assays and screening for factor inhibitors a retrospective study was performed on PTT 1:1 mix results on patients who also had LAC assays performed. The effects of LAC strength and length of incubation on PTT 1:1 mix prolongation were analyzed.

\section{MATERIALS AND METHODS}

All studies were performed by the Department of Laboratory Medicine at the University of Washington. PTT 1:1 mixing studies (PTT Automate, Diagnostica Stago, Asnieres, France) use equal portions of patient plasma and normal pooled plasma. The normal pooled plasma is tested before use to determine if it is within an acceptable normal range $(24-32$ seconds). Additional individual aliquots of the patient's plasma and normal pooled plasma are incubated along with the mix aliquot. At the end of the incubation period, a PTT is repeated on all three aliquots. The average normal pooled plasma immediate PTT is $29.14+/-0.65$ seconds and the average normal pooled plasma incubated PTT was $29.21+/-0.54$ seconds. Samples with PTT prolongation of the patient's incubated mix as compared to the patient's immediate mix of greater than 5 seconds were investigated further with a Bethesda (Coamatic factor VIII, Chromogenix, Bedford, MA) and hexagonal phase phospholipid assay (Staclot LA, Diagnostica Stago, Asnieres, France). 
PTT 1:1 mix incubations were originally performed at 1 hour in our laboratory. The incubation time was then extended to 2 hours to increase sensitivity for FVIII inhibitors in response to one case of a confirmed FVIII inhibitor which required a 2 hour incubation to detect.

A retrospective analysis was performed evaluating PTT 1:1 mix assays with incubation encompassing periods during which incubation was performed for either one hour (33 months total) or for two hours (7 months). Patients that had concurrent 1:1 mix and LAC assays were included in the study. In our laboratory, PTT prolongations are measured as the difference of the patient's incubated mix PTT as compared to the patient's immediate mix PTT as long as normal pool plasma PTT prolongation with incubation was less than 3 seconds (Table 1). A clotting time prolongation of 6-7 seconds (borderline) or $\geq 8$ seconds (positive) in the incubated PTT 1:1 mix was the criteria used to screen for possible FVIII inhibitors; most specimens were then retested by a FVIII inhibitor Bethesda assay. The residual factor VIII activity was measured using a chromogenic assay to eliminate possible interference from the LAC. Patients that screened positive for both the one and two hour incubation times were analyzed for true and false positives for FVIII inhibitor.

A second data analysis compared the strength of the LAC with prolongation of 1:1 mix clotting times. LAC strength was defined using hexagonal phase assay cutoffs of negative (NEG) $\leq 7$ seconds, borderline positive lupus (BLUP) 7.1 - 10.9 seconds, moderate positive lupus (MLUP) 11- 20 seconds and strong positive lupus (SLUP) $>20$ seconds. These groups were then stratified by 1 hour and 2 hour incubations.

\section{RESULTS}

Evaluation of the effect of incubation time on the sensitivity and specificity of FVIII screening by PTT 1:1 mix was performed. A total of 297 1:1 mixes with concurrent LAC tests were identified; 214 of these were incubated for 1 hour and 83 were incubated for 2 hours. Among all 1:1 mixes, only 1 was confirmed to have a FVIII inhibitor.

Within the 2 hour incubation group, 12 out of 83 patients screened positive $(14.5 \%)$ with clotting time prolongations $\geq 6$ seconds. Five of these cases had a $>8$ second prolongation and 7 cases were considered borderline with 6-7 second prolongation. None were found positive for FVIII inhibitor. Of the 12, 8 (67\%) were found to be positive for LACs (range 6-15 second prolongation), 1 was negative for both LAC and FVIII inhibitor, and 3 of the borderline group had no further tests done.

Within the 1 hour incubation group, 214 patients had concurrent PTT 1:1 mixes with LAC assays performed. Of those, 5 cases had $\mathrm{a} \geq 8$ second prolongation and 2 cases were considered borderline with 6-7 second prolongations giving a total of 7 samples which screened possible for FVIII inhibitors (2.3\%). Of these, 3 were found to be positive for a strong LAC, 1 was positive for a moderate LAC and 1 was positive for a FVIII inhibitor (13 second prolongation). The 2 patients in the borderline group had no further tests done.

This results in an $80 \%$ false positive screen for FVIII inhibitor with the $\geq 8$ second prolongation in the 1 hour incubation group as compared to a $100 \%$ false positive rate in the $>8$ second prolongation in the 2 hour incubation group. In conclusion, the $2 \mathrm{hr}$ incubation had more false positives for FVIII screen which can be largely attributed to the presence of LACs. In summary, in the $>8$ second prolongation group, $80 \%$ were false positive ( 5 of 7 ) in the 1 hour incubation group while $100 \%$ ( 5 of 5 ) of the 2 hour incubation group were false positive. Almost all of the false positive tested could be attributed to the presence of LAC.

When prolongation times were compared to strength of LAC (Figure 1A) there was an increase of average prolongation (seconds) when compared with LAC strength (NEG, $1 \pm 0.3$; BLUP, $2 \pm 0.6$; MLUP, $3 \pm 0.5$; SLUP, $5 \pm 1$ standard error of the mean (SEM)). This trend of prolongation correlates with the strength of lupus inhibitor ( $\mathrm{p}=0.002$, one-way ANOVA). No significant difference was found between NEG and borderline (BLUP) LAC groups and no significant difference was found between moderate (MLUP) and strong (SLUP) LAC groups by Student's $t$-test analysis. Though there is also no statistical difference between BLUP and MLUP ( $\mathrm{p}=0.3$ ), a decision was made to define two groups: NEG/BLUP and MLUP/SLUP since statistical significance was found between NEG and MLUP ( $\mathrm{p}=0.004)$ and between BLUP and SLUP $(\mathrm{p}=0.04)$. A significant difference was found between the NEG/BLUP groups combined when compared with 
the MLUP/SLUP groups combined (Figure $1 \mathrm{~B} ; \mathrm{p}=$ $0.0004)$. When the MLUP/SLUP group was stratified for incubation times of 1 and 2 hours, the prolongation of PTT was significantly increased with the longer incubation (Figure 2; Student's $t$-test, two tailed; $\mathrm{p}=$ $0.009)$. There is no statistical difference between 1 hour and 2 hour incubated NEG/BLUP groups ( $\mathrm{p}=0.4$ ).

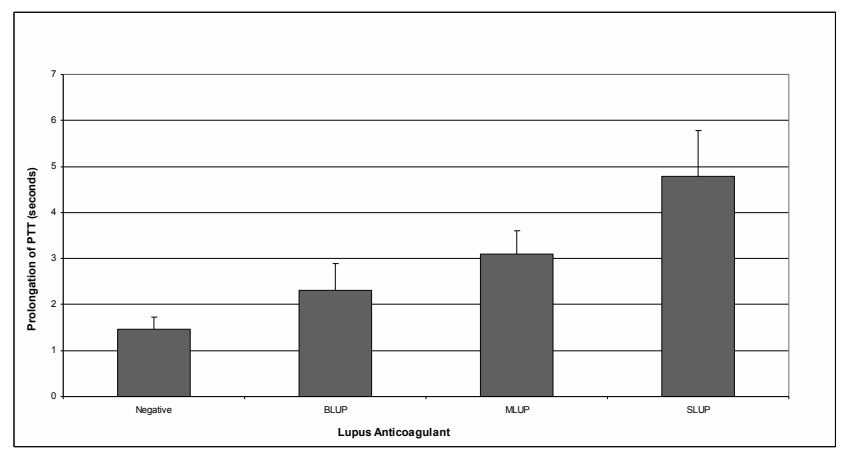

Figure 1A. There is a significant increasing trend of average PTT 1:1 mix prolongation with increasing LAC strength. One way ANOVA, $\mathrm{p}=0.002$.

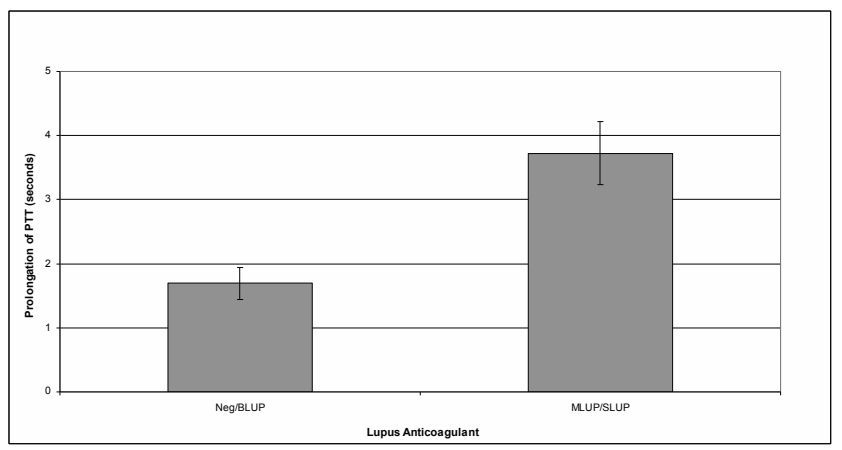

Figure 1B. A significant difference in PTT 1:1 mix prolongation is found when comparing NEG/BLUP combined group to MLUP/SLUP combined group. Student's $t$-test, 2tailed; $\mathrm{p}=0.0004$. No significant difference is found between NEG and BLUP or between MLUP and SLUP. NEG = negative, $\mathrm{BLUP}=$ borderline, $\mathrm{MLUP}=$ moderate, SLUP = strong lupus anticoagulant.

\section{DISCUSSION}

The presence of a moderate or strong LAC can cause a significantly increased prolongation of PTT 1:1 mix after incubation when compared to PTT 1:1 mix in the absence of LAC. This prolongation showed a significant positive correlation with increasing LAC strength. This effect was significantly amplified by increasing incubation time from 1 hour to 2 hours.

Our data showed using longer incubation times for PTT 1:1 Mix for Factor VIII inhibitor screening results in more false positives, mostly due to LACs. This phenomenon is not rare as we observed $14.5 \%$ false positive rate with PTT prolongations $\geq 6$ seconds using the 2 hour 1:1 mix incubation. Consequently, the increase in false positives caused our laboratory to return to using a 1 hour incubation. LACs are fairly common, present in $>50 \%$ of critically ill patients. ${ }^{8}$ Therefore, it is important to consider the effect of a possible LAC on the interpretation of the PTT prolongation and to be aware that this effect is influenced by both incubation time and strength of LAC.

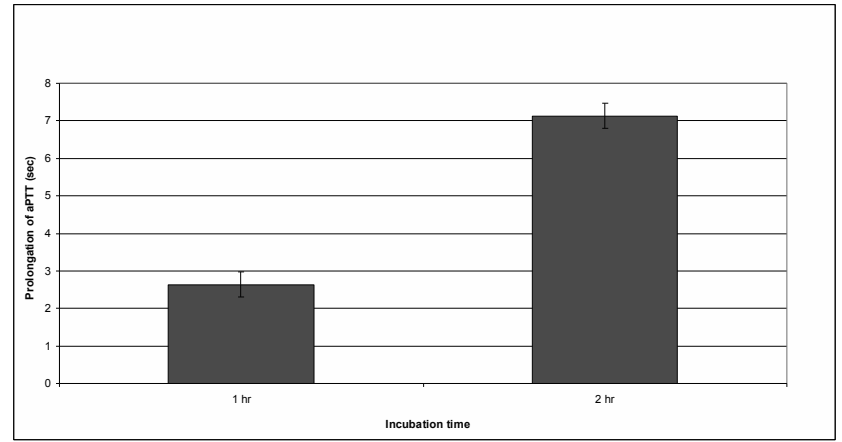

Figure 2. In samples positive for LAC, a 2 hour incubation of 1:1 Mix causes a significantly increased prolongation of PTT when compared to a 1 hour incubation. Student's $t$-test, 2 -tailed; $\mathrm{p}=0.009$. MLUP $=$ moderate and SLUP $=$ strong lupus anticoagulant.

The mechanism of LAC interference of clotting assays has yet to be determined. As our data shows, prolongation of the PTT 1:1 mix increases with incubation time. This suggests interaction of the LAC with phospholipids may be time and temperature dependent. Alternatively, during incubation LAC may form complexes with other proteins, such as $\beta 2$ glycoprotein I and/or cardiolipin with higher affinity for phospholipid. ${ }^{2,9}$ These incubated complexes may be better able to compete for phospholipids causing a prolongation in the PTT.

\section{ACKNOWLEDGMENTS}

We will like to thank Dr. Noah Hoffman for his expertise and assistance for the acquisition of the data from the LIS system. We'd also like to thank Sara Trimble for her editorial comments and suggestions.

\section{REFERENCES:}

1. Mendoza-Pinto C, Garcia-Carrasco M, Jimenez-Hernandez M, et al. Etiopathogenesis of Behcet's disease. Autoimmun Rev. Feb 2010;9(4):241-5.

2. Triplett DA. Antiphospholipid antibodies. Arch Pathol Lab 
Med. Nov 2002;126(11):1424-9.

3. Verbruggen B, van Heerde WL, Laros-van Gorkom BA. Improvements in factor VIII inhibitor detection: From Bethesda to Nijmegen. Semin Thromb Hemost. Nov 2009;35(8):752-9.

4. Miyakis S, Lockshin MD, Atsumi $\mathrm{T}$, et al. International consensus statement on an update of the classification criteria for definite antiphospholipid syndrome (APS). J Thromb Haemost. Feb 2006;4(2):295-306.

5. Eby C. Antiphospholipid syndrome review. Clin Lab Med. Jun 2009;29(2):305-19.

6. Pengo V, Tripodi A, Reber G, et al. Update of the guidelines for lupus anticoagulant detection. Subcommittee on Lupus Anticoagulant/Antiphospholipid Antibody of the Scientific and
Standardisation Committee of the International Society on Thrombosis and Haemostasis. J Thromb Haemost. Oct 2009;7(10):1737-40.

7. Tripodi A. Laboratory testing for lupus anticoagulants: diagnostic criteria and use of screening, mixing, and confirmatory studies. Semin Thromb Hemost. Jun 2008;34(4):373-9.

8. Wenzel C, Stoiser B, Locker GJ, et al. Frequent development of lupus anticoagulants in critically ill patients treated under intensive care conditions. Crit Care Med. Apr 2002;30(4):76370.

9. de Groot PG, Derksen RH. The antiphospholipid syndrome: clinical characteristics, laboratory features and pathogenesis. Curr Opin Infect Dis. Jun 2005;18(3):205-10.

\section{ASCLS Annual Meeting Sponsors}

\section{ASCLS gratefully acknowledges the following companies for their generous financial} support of the 80th Annual Meeting, July 17-21, Los Angeles, CA

\section{REGISTRATION PORTFOLIOS \\ Roche Diagnostics \\ CLOSING SESSION \\ Siemens Healthcare Diagnostics \\ INTERNET CAFÉ \\ Abbott Diagnostics}

MOBILE MEETING APPLICATION

Bio-Rad Laboratories, Inc.

PRELIMINARY PROGRAM

The Binding Site

THURSDAY PLENARY

Carolina Liquid Chemistries Mindray

\section{SCIENTIFIC SESSIONS}

ADVANCE Newsmagazines American Red Cross Blood Services American Society for Clinical Pathology

ARUP Laboratories

Beckman Coulter, Inc.

CellaVision $\mathrm{AB}$

DRG International, Inc.

Hardy Diagnostics

Immco Diagnostics, Inc.

McKesson Provider Technologies

Medical Chemical Corporation

Quest Diagnostics

TerumoBCT 